\title{
LA MESURE DE LA PRODUCTION LAITIÈRE CHEZ LA TRUIE
}

PAR

E. SALMON LEGAGNEUR ( 1 )

Station de Recherches sur 1'Élevage, C.N.R.Z., Jouy-en-Josas

\section{I. - HISTORIQUE}

Les premiers essais de détermination de la production laitière d'une truie remontent à 1865 . Ils sont dus à Von GoHren qui, par pesée de porcelets avant et après des tétées espacées de 3 heures, estima à $I, 54 \mathrm{~kg}$ la quantité de lait produite par jour par une truie à sa quatrième semaine de lactation.

Depuis cette date, de nombreuses autres mesures furent effectuées par des chercheurs de différents pays. Ces mesures relèvent toutes de l'une des trois méthodes suivantes :

\section{I $^{0}$ Appréciation de la lactation à partir de la croissance des porcelets}

Cette méthode est sans doute la moins précise, elle bénéficie encore d'une certaine faveur en raison de sa facilité d'exécution.

Elle repose sur l'existence d'une corrélation assez étroite entre la croissance des porcelets pendant une période donnée, ou leur poids à certains stades (3 semaines, I mois, sevrage), et la quantité de lait maternel qu'ils ont reçu pendant cette période ou jusqu'à ce stade. La connaissance $d u$ poids des porcelets donnerait donc un bon indice de la production laitière des truies.

Selon S. Bengtsson (I923-24), des auteurs danois furent sans doute les premiers, aux environs de I900, à utiliser cette méthode. Mais, méconnaissant la valeur énergétique du lait de truie, ils évaluèrent à $20 \mathrm{~kg}$ de lait la production quotidienne d'une truie dont la portée croissait de $I, 8 \mathrm{~kg}$ par jour.

Les mesures effectuées par K. M. ANDERSEN en I924 donnèrent

(1) Avec la collaboration technique de J. Rettagliati.

Annales de Zootechnie. - I956. 
un résultat plus vraisemblable, quoique probablement encore trop élevé. Selon lui, la quantité de lait nécessaire chaque jour à une portée de Io porcelets était en moyenne de $\mathrm{I} 2 \mathrm{~kg}$.

Plus récemment, A. M. LEROY (I937) estima qu'il était possible, en connaissant le croît journalier et le nombre des porcelets d'une portée, d'en déduire la production laitière approximative de la truie. I1 donne à ce sujet le tableau suivant :

\section{TABLEAU I}

Variations de la production laitière des truies en fonction de la vitesse de croissance des porcelets (d'après A. M. I,EROY).

\begin{tabular}{c|c|c|c|c}
\hline \hline $\begin{array}{c}\text { Poids moyen d'un } \\
\text { porcelet a l'âge } \\
\text { de 2I jours }\end{array}$ & $\begin{array}{c}\text { Gain de poids } \\
\text { journalier d'un } \\
\text { porcelet }\end{array}$ & \multicolumn{3}{|c|}{ Quantité de lait à $7,5 \%$ de M.G. (par jour) } \\
\cline { 2 - 4 } & I00 & 6 porcelets & 8 porcelets & 10 porcelets \\
$3,1 \mathrm{~kg}$ & 150 & $3, \mathrm{I}-4,2 \mathrm{~kg}$ & $3,4-4,6 \mathrm{~kg}$ & $3,6-4,9 \mathrm{~kg}$ \\
4,3 & 200 & $4,3-4,9$ & $4,7-5,4$ & $5,0-5,8$ \\
5,4 & $5,3-6,4$ & $5,8-7,0$ & $6,2-7,5$ \\
6,5 & 250 & $6,8-8,0$ & $7,5-8,8$ & $8,0-9,4$ \\
\hline
\end{tabular}

B. Navratil (r955), enfin, utilise le poids de la portée à 2 I jours comme base d'appréciation de la valeur laitière des truies. Il reconnaît toutefois que la méthode est sujette à des erreurs pouvant atteindre 22 p. Ioo des estimations ainsi faites.

D’une manière générale, on peut reprocher à ces méthodes, outre leur manque de précision :

Io De ne pas tenir compte des différences parfois considérables existant dans l'utilisation nutritive du lait par les porcelets des différentes portées.

$2^{\circ}$ De donner des indications généralement valables pour la seule première partie de la lactation (porcelets de moins d'un mois).

\section{$2^{\circ}$ Mesure de la quantité de lait produite par les truies, par la détermination de leurs pertes de poids pendant les tétées}

Cette méthode a été utilisée par H. CLAUSEN et ses collaborateurs (I952). Elle consiste à placer la truie à contrôler sur une loge-bascule permettant de connaître le poids de la truie à un instant donné.

Par pesée de la truie immédiatement avant et après les tétées, on peut connaître les quantités de lait consommées par les porcelets. Ces quantités de lait servent de point de départ au calcul des productions laitières journalières, puis de l'ensemble de la lactation. 
Des mesures ainsi faites sur $I_{3}$ truies ayant une moyenne de ro porcelets, donnèrent les résultats suivants :

\section{TABLEAU II}

Variations de la production de 13 truies au cours de la lactation

\begin{tabular}{|c|c|c|c|c|c|c|c|c|}
\hline $\begin{array}{c}\text { Nombre de jours après la mise- } \\
\text { bas } \ldots \ldots \ldots \ldots \ldots \ldots \ldots \ldots \ldots\end{array}$ & 3 & 10 & I7 & 24 & $3^{\text {I }}$ & 38 & 45 & $5^{2}$ \\
\hline $\begin{array}{l}\text { Production laitière journalière } \\
(\mathrm{kg}) \quad \ldots \ldots \ldots \ldots \ldots \ldots \ldots \ldots\end{array}$ & 6,87 & 7,83 & 8,03 & 7,88 & 8,27 & 7,77 & 7,36 & 6,28 \\
\hline
\end{tabular}

Cette méthode semble pouvoir donner des résultats intéressants. Nous lui reprocherons cependant d'être assez peu précise, par suite des erreurs dues aux opérations de pesée de la truie.

Il semble très difficile de connaitre le poids d'une truie avec une précision supérieure à $50 \mathrm{~g}$, car on est limité dans ce domaine :

I $^{\circ}$ Par la sensibilité des balances,

$2^{\circ}$ par les mouvements imperceptibles et la respiration des truies qui rendent illusoires les lectures de poids faites à une précision supérieure.

La mesure nécessitant deux pesées, l'erreur possible est donc de l'ordre de $\mp$ roo g. Comme la quantité de lait produite au cours de la tétée varie généralement de 100 à $500 \mathrm{~g}$, l'erreur commise peut atteindre 20 à 50 p. Ioo de la valeur mesurée.

Une comparaison des résultats obtenus par cette méthode et par la méthode que nous proposons plus loin, a été effectuée. Nous avons fait porter cette comparaison sur 4 tétées contrôlées simultanément par les deux procédés. On a trouvé les résultats suivants :

TABLEAU III

\begin{tabular}{|c|c|c|c|c|}
\hline \multirow{2}{*}{ Tétées } & \multicolumn{2}{|c|}{ Contrôle par pesée de la truie } & \multicolumn{2}{|c|}{ Méthode proposée } \\
\hline & Lait $g$ & Précisiong $g$ & Lait $g$ & Précision $\mathrm{g}$ \\
\hline $\begin{array}{c}\text { I } \\
2 \\
3 \\
4 \\
\text { Moyenne }\end{array}$ & $\begin{array}{l}650 \\
350 \\
525 \\
625 \\
540\end{array}$ & $\begin{array}{l}\frac{ \pm}{ \pm} 100 \\
\pm \| \\
\pm \| \\
\pm \quad " \\
\pm 100 \\
\pm 100\end{array}$ & $\begin{array}{l}505 \\
355 \\
450 \\
530 \\
460\end{array}$ & $\begin{array}{c} \pm 20 \\
" \\
" \\
\pm " \\
\pm 20\end{array}$ \\
\hline
\end{tabular}

$3^{\circ}$ Mesure de la quantité de lait produite par les truies, par l'évaluation du gain de poids de la portée au cours des tétées

Cette méthode est la plus employée et il semble que ce soit celle qui donne lieu aux déterminations les plus précises.

Parmi les auteurs qui 1'utilisèrent, citons: Von GoHren (I865), 
Henry et Wohl (I897), Carlyle (I903), Schmidt et Lauprecht (I926), HeMPEL (I928), WOHLBIER (I929), OlOFSSON (I930), ThOMPSON (I93I), Hughes et Hart' (I935), Bonsma (I935), Donald (I936), DschapaRIDSE (I936), WeLLLS (I940), Filmer (I949), Niwa (I95I), LALEVIC (I953), SMith (I950, I952, I955), BERGE et INDREBo (I953), BARBER et BRAUDE (I955).

Le principe de la mesure est voisin de celui de la méthode précédente : on pèse les porcelets avant et après les tétées, et la différence entre les deux poids représente la quantité de lait que la truie a donné à sa portée.

On trouvera au tableau IV un aperçu des résultats ainsi obtenus par différents chercheurs.

TABLEAU IV

\begin{tabular}{|c|c|c|c|c|c|c|c|}
\hline Auteur & Année & $\begin{array}{c}\text { Nombre } \\
\text { de lacta- } \\
\text { tions }\end{array}$ & $\begin{array}{l}\text { Intervalle } \\
\text { entre les } \\
\text { contrôles }\end{array}$ & Jours de contrôle & 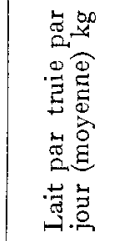 & 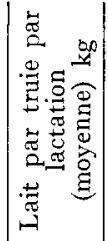 & 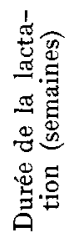 \\
\hline Von GoHren & 1865 & I & $3 h$ & $4^{e}$ semaine & $\mathrm{I}, 54$ & - & - \\
\hline HENRY \& W & 1897 & 4 & $2-4 \mathrm{~h}$ & $3 j$ tous les $17 j$ & 2,4 & 165 & To \\
\hline CARLYLE ... & 1903 & 12 & $2-4 \mathrm{~h}$ & $2 \mathrm{j}$ I fois par mois & 2,83 & 210 & $\mathrm{r} 2$ \\
\hline & & $3^{6}$ & & I j par semaine & 3,15 & & $\mathrm{x} 2$ \\
\hline WOHLBIER & 1928 & 4 & Ih 30 & I j par semaine & 4,8 & - & 4 \\
\hline HEMPEL $\ldots \ldots \ldots \ldots$ & I928 & 22 & $3 \mathrm{~h}$ & I ju par semaine & 3,32 & - & \\
\hline OLOFSSON \& LARSON & & 4 & $\mathrm{I}-2 \mathrm{~h}$ & par semaine & 4,8 & 270 & 8 \\
\hline HUGHES \& HART $\ldots$ & 193 & 2 & $2-4-6 h$ & $\mathrm{I} j$ tous les $\mathrm{ioj}$ & 3,5 & 242 & Io \\
\hline BONSMA...... & 193 & $5^{2}$ & $2 \mathrm{~h}$ & I j par semaine & & $3^{6} 7$ & 8 \\
\hline DSCHAPARIDS & 1936 & & & & $2,09-5,19$ & & \\
\hline WELLS \& BEESON & & 43 & $I-2 h$ & I j par semaine & 5,3 & - & 8 \\
\hline Filmer $\ldots \ldots \ldots$ & & 5 & & I j par semaine & & 274 & \\
\hline Niwa . & 19 & 9 & I $-\mathbf{I} h 3^{\circ}$ & I j par semaine & 4,2 & 254 & 8,5 \\
\hline Sмiтн $\ldots \ldots \ldots \ldots \ldots$ & $195^{\circ}$ & 5 & $\mathbf{I ~ h}$ & I j par semaine & & $|\mathrm{I} 36-430|$ & 8 \\
\hline & 1952 & 15 & $\mathrm{I}-\mathrm{I}$ h $\mathrm{I} 5$ & I $2 \mathrm{~h}$ par semaine & ניב & 360 & 8 \\
\hline BERGE & $195^{2}$ & 28 & I h & 2 fois $4 \mathrm{~h}$ parse- & $6,05-7,47$ & & 8 \\
\hline Barber \& BraUde $\ldots$ & 1955 & 3 & I h & I j par semaine & $6,2 \mathrm{I}$ & 348 & 8 \\
\hline
\end{tabular}

Les différences assez considérables que l'on peut observer entre ces résultats semblent provenir en partie des modalités particulières de contrôle propres à chaque auteur.

Ces modalités ont trait principalement aux points suivants :

I $^{0}$ Conduite des opérations au cours des mesures,

$2^{0}$ intervalles entre les tétées,

$3^{o}$ nombre de mesures nécessaires pour connaitre la production journalière,

$4^{\mathbf{0}}$ nombre de jours de contrôle pour connaître la production laitière d'une truie sur toute sa lactation.

Dans l'étude qui va suivre, nous avons repris chacun de ces points, 
afin de déterminer le plus rationnellement possible la règle d'emploi de cette méthode.

\section{II. - LES OPÉRATIONS DE MESURE}

Nous avons choisi de peser les porcelets par portée entière (ou à défaut par demi-portée), comme l'avaient fait SMITH (1952) et BERGE et INDREBo (I953), plutôt qu'individuellement comme l'avaient fait Donald (I936) ou BARBER et Braude (I955).

Nous avons estimé que cette méthode était préférable, car elle permet de limiter au maximum le temps passé entre la pesée avant et la pesée après la tétée. On élimine ainsi une partie des erreurs inhérentes au contrôle.

Le problème de la sensibilité de la balance à utiliser nous a semblé être le point le plus délicat : nous avons utilisé une balance d'une précision de \pm Io $\mathrm{g}$ pour une portée de $140 \mathrm{~kg}$. Cette balance est du type semi-automatique à double pendule de compensation et levier de tare ; son cadran est gradué de o à $2 \mathrm{~kg}$ par divisions de $20 \mathrm{~g}$ (cliché $\mathrm{I}$ ).

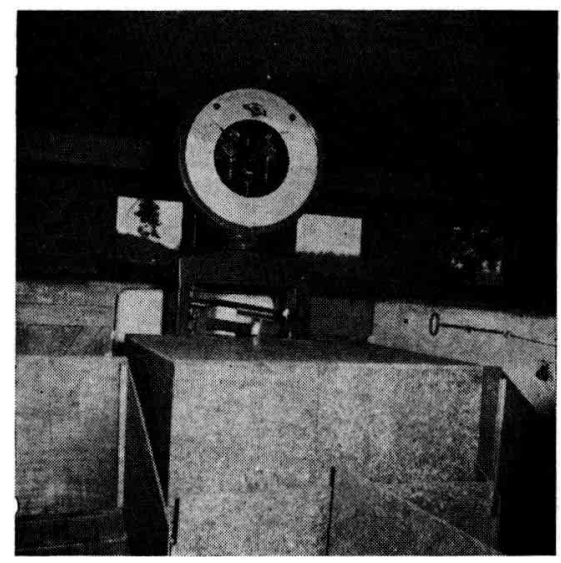

Cliché I

L'amortissement des oscillations ell cours de pesée est assuré par un double frein à huile et par un dispositif de limitation des oscillations. Lorsque les porcelets sont très agités, la lecture des poids sur le cadran de la balance peut cependant être difficile.

Le plateau de la balance est équipé d'une caisse de $\mathrm{I} \mathrm{m} \times 0,9 \mathrm{~m}$ $\times 0,6 \mathrm{~m}$ dont le volume intérieur est réglable en fonction du nombre et de 1'âge des porcelets à peser.

Un dispositif d'amenée des porcelets permet de faire monter et descendre ces derniers dans la caisse sans a voir à les manipuler (clichés 2, 3 et 4$)$. 
I a balance est placée au centre d'une case abritant deux portées de porcelets. Des trappes de chaque côté de la case permettent de faire passer les porcelets dans les loges des truies au moment des tétées (fig. I).

La succession des opérations de contrôle est la suivante :

I $^{\circ}$ Les porcelets sont réveillés et conduits sur une aire de ciment

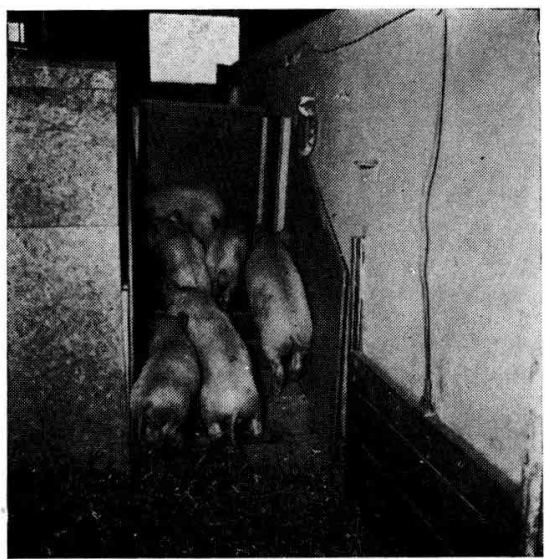

Cliché 2

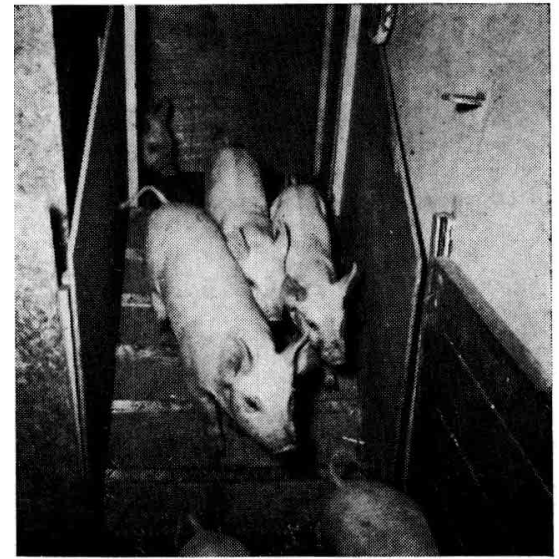

Cliché 3

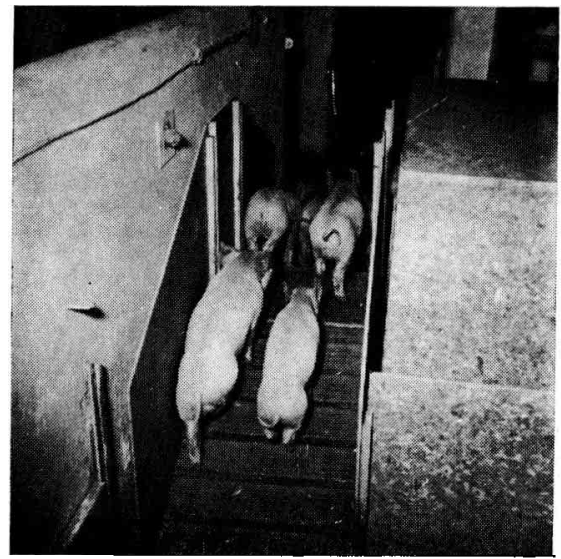

Cliché 4

froid pour les inciter à uriner. Cette opération a pour but d'éviter les mictions et les défécations entre deux pesées;

$2^{0}$ les porcelets montent sur la bascule et sont pesés. Si le poids de la portée est supérieur à $130 \mathrm{~kg}$ (fin de lactation), la portée est fractionnée en deux lots pesés séparément; Après pesée, ils retournent avec la truie.

$3^{\circ}$ la tétée a lieu sous surveillance;

$4^{\circ}$ les porcelets sont repesés;

$5^{0}$ les porcelets se recouchent. 
La durée totale des opérations varie de ro à $\mathrm{I}_{5}$ minutes. Le temps qui s'écoule entre les deux pesées est d'environ 3 à 4 minutes.

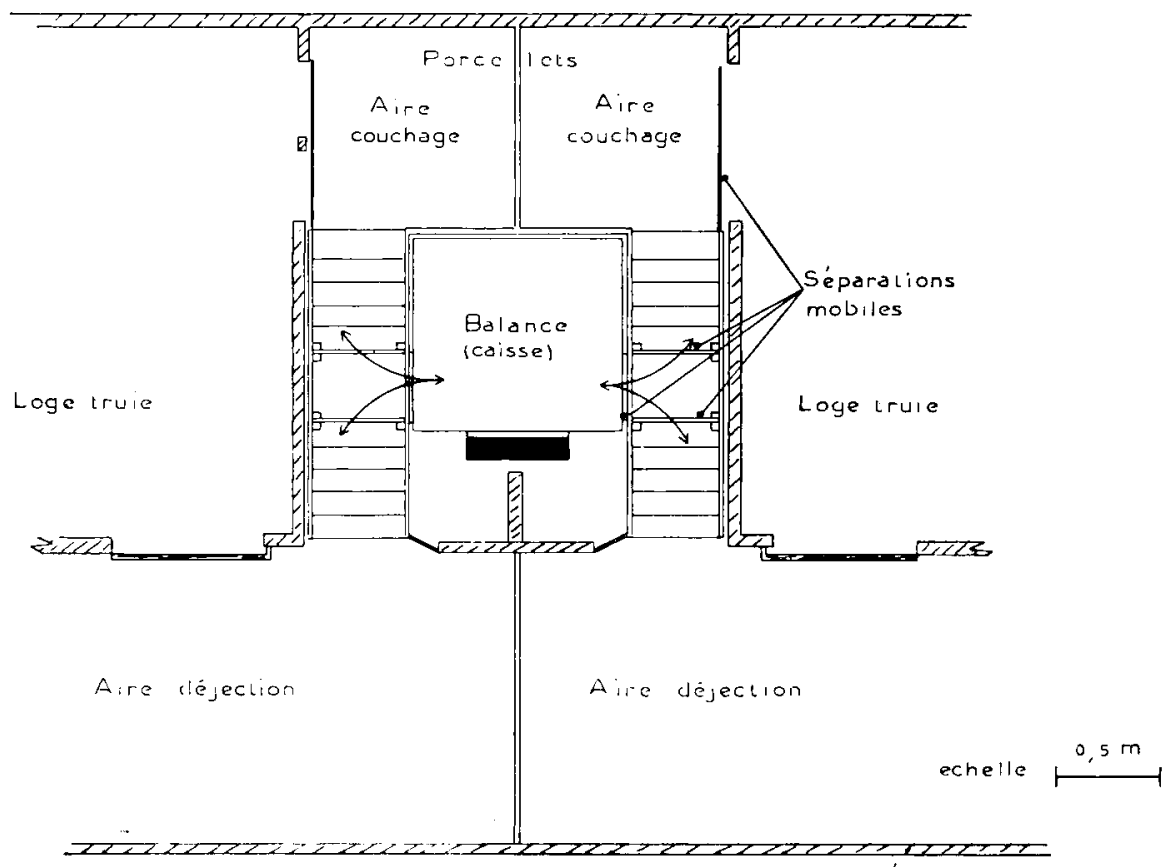
Fig. I. - Aménagement d'une loge permettant le contrôle simultané de la production laitière

\section{III. - INTERVALLE ENTRE LES TÉTÉES}

L'observation courante montre que c'est généralement la truie qui appelle ses petits au moment des tétées et que celles-ci semblent régulièrement espacées. Il peut arriver cependant que, sollicitée par les porcelets, la truie accepte de se laisser téter. Il faut toutefois que ces sollicitations se fassent à un rythme voisin de celui consenti par la truie.

Or, pour pouvoir procéder commodément aux opérations de contrôle, il est nécessaire de séparer la truie de ses porcelets et de ne les remettre en présence qu'au moment choisi pour la mesure.

Il importait donc d'imposer aux mesures une cadence acceptée à la fois par la truie et par les porcelets.

Les premiers chercheurs avaient utilisé des intervalles variables de 2-3-4 ou 5 heures. Or, ces intervalles nous semblent aujourd'hui très supérieurs à 1'intervalle naturel imposé par la truie.

Comme l'ont montré Olofisson (I930), puis WeLLS et BeEsoN (I940), l'intervalle choisi pour les tétées a une influence sur la quantité totale de lait produit au cours de la journée. On chercha donc à se rapprocher davantage de la cadence naturelle de la truie. 
J. H. SHEPPERD (I929) avait montré que l'intervalle moyen séparant 2 tétées chez le porc était d'environ 62 minutes (maximum I75 minutes).

S. BERGE et $\mathrm{T}$. INDREBO (I953), analysant la distribution des tétées de quatre portées sur un intervalle de 24 heures, conclurent que le meilleur intervalle à utiliser pour les mesures de la production laitière des truies semblait être de 60 minutes.

D. M. Smith (I952) fait remarquer que l'intervalle entre les tétées varie suivant les truies et leur stade de lactation. Il propose de déterminer, avant chaque jour de contrôle, l'intervalle à adopter en se basant sur le nombre de tétées du jour précédent. L'intervalle moyen qu'il utilise est voisin de I heure.

R. S. BARBER et R. Braude (I954) vérifient que de grands intervalles (2-3 heures) conduisent à une sous-estimation de la production laitière, car la truie produit alors moins de lait, se fatigue davantage et les porcelets perdent du poids au lieu d'en gagner au cours de la journée de contrôle. Ils préconisent également des contrôles espacés d'une heure, mais reconnaissent que, dans quelques cas, les truies peuvent refuser de se laisser téter.

Nous avons, pour notre part, essayé d'adopter l'intervalle moyen de 60 minutes dans nos mesures, mais une assez forte proportion d'échecs nous a conduit à reconsidérer le problème des fréquences naturelles des tétées.

Vingt une truies, à des stades variés de lactation, furent mises en observation jour et nuit pendant 24 heures. Les intervalles entre tétées furent chaque fois notés et permirent de calculer les valeurs suivantes :

Intervalle moyen des tétées (24 heures) $=65,5$ minutes $\pm 20,6$.

Intervalle moyen des tétées de jour $(6 \mathrm{~h}$. du matin à $6 \mathrm{~h}$. du soir $)$ $=65,0$ minutes $\pm 20,5$.

Intervalle moyen des tétées de nuit ( $6 \mathrm{~h}$. du soir à $6 \mathrm{~h}$. du matin) $=66,0$ minutes $\pm 20,6$.

L'intervalle minimum observé fut de 22 minutes et le plus long, de I33. Le tableau $\mathrm{V}$ montre en outre la répartition des intervalles observés, classés suivant leur durée.

TABLEAU V

Intervalles entre tétées (répartition)

\begin{tabular}{|c|c|c|c|c|c|c|c|}
\hline Durée (minutes) $\ldots \ldots \ldots$ & $10-25$ & $25-35$ & $35-45$ & 45 & -55 & $55-65$ & $65-75$ \\
\hline $\begin{array}{l}\text { Nombre............ } \\
\% \ldots \ldots \ldots \ldots\end{array}$ & $\begin{array}{l}4 \\
\mathbf{I}\end{array}$ & $\begin{array}{l}16 \\
4,2\end{array}$ & $\begin{array}{l}47 \\
12,4\end{array}$ & & $\begin{array}{l}69 \\
18,2\end{array}$ & $\begin{array}{l}84 \\
22, \mathrm{I}\end{array}$ & $\begin{array}{l}6 \mathrm{I} \\
\mathrm{I} 6, \mathrm{I}\end{array}$ \\
\hline Durée (minutes) . . . . . & $75-85$ & $85-95$ & $95-$ & 105 & 105 & - II5 & $>I{ }_{5}$ \\
\hline 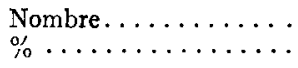 & $\begin{array}{l}3^{8} \\
\text { 10 }\end{array}$ & ${ }^{23} 6,1$ & $\begin{array}{l}2 \mathrm{I} \\
5,5\end{array}$ & & & $\begin{array}{l}2 \\
3,2\end{array}$ & $\begin{array}{l}4 \\
\mathbf{r}, \mathbf{I}\end{array}$ \\
\hline
\end{tabular}


Par ailleurs, en fonction du stade de lactation, les intervalles moyens observés ont été les suivants :

\author{
TABLEAU VI \\ Variations de l'intervalle entre tétées \\ avec le stade de lactation
}

\begin{tabular}{|c|c|c|}
\hline Stade de lactation & Nombre de truies & Intervalle moyen (minutes) \\
\hline $\begin{array}{l}\mathrm{I}^{\mathrm{re}}-\mathbf{2}^{\mathrm{e}} \text { semaine } \ldots \ldots \ldots \ldots \ldots \ldots \\
3^{\mathrm{e}}-4^{\mathrm{e}} \text { semaine } \ldots \ldots \ldots \ldots \ldots \ldots \ldots \\
5^{\mathrm{e}}-6^{\mathrm{e}} \text { semaine } \ldots \ldots \ldots \ldots \ldots \ldots \\
7^{\mathrm{e}}-\mathrm{\delta}^{\mathrm{e}} \text { semaine } \ldots \ldots \ldots \ldots \ldots \ldots\end{array}$ & $\begin{array}{l}4 \\
9 \\
3 \\
5\end{array}$ & $\begin{array}{l}67 \\
61 \\
70 \\
74\end{array}$ \\
\hline
\end{tabular}

Il semble, à l'appui de ces deux séries de résultats :

I $^{\circ}$ que l'intervalle moyen entre les tétées soit nuit, et jour, voisin de 65 minutes,

$2^{0}$ que cet intervalle varie peu au cours de la lactation ( il augmente légèrement en fin de lactation),

$3^{\circ}$ qu'il varie, par contre, assez fortement au cours de la journée et avec les truies.

En fonction de cette dernière observation, nous avons été conduits à adopter pour nos mesures un intervalle constant de 75 minutes $(65$ minutes plus ro minutes de marge de sécurité pour tenir compte des variations individuelles). Cet intervalle nous a donné satisfaction dans la presque totalité des cas et au cours de toute la lactation.

\title{
IV. - NOMBRE DE MESURES PAR JOUR
}

Il est évident que la méthode de référence de la mesure de la production laitière journalière exige le contrôle de toutes les tétées, de jour comme de nuit, pendant 24 heures, ce qui astreint à un travail considérable.

En fait, l'égalité des intervalles entre les tétées de nuit et de jour laisse supposer que les productions de lait correspondantes pourraient être très voisines.

De ce fait, quelques auteurs utilisèrent la seule production diurne ( $6 \mathrm{~h}$. du matin $-6 \mathrm{~h}$. du soir) pour le calcul de la production par 24 heures.

BERGE (I955) utilisa même la production de 4 heures $(8 \mathrm{~h}$. - I $2 \mathrm{~h}$.) mesurée deux jours de suite et obtint la production journalière en multipliant par 6 la moyenne de ces deux valeurs.

Nous avons donc cherché :

$I^{0}$ à évaluer la différence de production pouvant exister chez un même animal le jour et la nuit,

$2^{0}$ à suivre les variations de cette production au cours de la journée. 


\section{$\mathrm{I}^{0}$ Production du jour et production de la nuit}

Huit truies furent contrôlées pendant 24 heures (à raison d'une mesure toutes les 75 minutes). Les résultats furent les suivants :

TABLEAU VII

\begin{tabular}{|c|c|c|c|}
\hline Truies & Production diurne & Production nocturne & P. nuit - P. jour \\
\hline $\begin{array}{l}868 \\
885 \\
868 \\
885 \\
411 \\
083 \\
083 \\
086\end{array}$ & $\begin{array}{l}3060 \mathrm{~g} \\
5 \text { I } 25 \\
4485 \\
4585 \\
2730 \\
3715 \\
2735 \\
2735\end{array}$ & $\begin{array}{l}3295 \mathrm{~g} \\
4750 \\
33865 \\
4445 \\
3250 \\
4275 \\
2190 \\
3 \text { I } 25\end{array}$ & $\begin{array}{l}+235 \mathrm{~g} \\
+375 \\
-620 \\
-140 \\
+\quad 520 \\
+560 \\
+\quad 45 \\
+\quad 390\end{array}$ \\
\hline Moyenne & $35^{84}$ & 3649 & $+65,6$ \\
\hline
\end{tabular}

La différence observée entre la production diurne et la production nocturne n'est pas significative ( $t=0,43$ pour 8 observations); elle est en moyenne de :

$$
65,6 \mathrm{~g} \pm 305 \mathrm{~g} \text {. }
$$

Ce résultat confirme qu'il serait donc possible de n'effectuer le contrôle de la lactation que sur I2 heures et de multiplier par deux le résultat pour avoir la production de 24 heures.

L'erreur maximum (seuil $\mathrm{P}=0,05$ ) sur la production de 24 heures obtenue par cette méthode a été calculée. E,lle est, en valeur absolue, de $506 \mathrm{~g}$.

\section{$2^{0}$ Variations de la production au cours de la journée}

Le tableau VIII donne la répartition par tranches de 6 heures des productions sur 24 heures des 8 truies précédentes.

$$
\text { TABLEAU VIII }
$$

Variations de la production laitière des truies

\begin{tabular}{|c|c|c|c|c|}
\hline Truies & $6 \mathrm{~h}-12 \mathrm{~h}$ & $12 h-18 h$ & $18 \mathrm{~h}-24 \mathrm{~h}$ & $24 \mathrm{~h}-6 \mathrm{~h}$ \\
\hline $\begin{array}{l}868 \\
885 \\
868 \\
885 \\
411 \\
083 \\
083 \\
086\end{array}$ & $\begin{array}{ll}\text { I } & 5 \mathrm{IO} \mathrm{g} \\
2 & 390 \\
2 & 315 \\
2 & 260 \\
\text { I } & 390 \\
\text { I } & 830 \\
\text { I } & 140 \\
\text { I } & 460\end{array}$ & $\begin{array}{ll}\text { I } & 550 \\
2 & 735 \\
2 & \text { I } \\
2 & 0 \\
2 & 325 \\
\text { I } & 340 \\
\text { I } 885 \\
\text { I } 095 \\
\text { I } 275\end{array}$ & $\begin{array}{l}\text { I } 580 \mathrm{~g} \\
2470 \\
2040 \\
2220 \\
\text { I } 610 \\
2210 \\
\text { I } 060 \\
\text { I } 55^{\circ}\end{array}$ & $\begin{array}{ll}\text { I } & 715 \mathrm{~g} \\
2 & 280 \\
\text { I } & 825 \\
2 & 225 \\
\text { I } & 640 \\
2 & 065 \\
\text { I } & 130 \\
\text { I } & 575\end{array}$ \\
\hline $\begin{array}{c}\text { Total } \\
\% \text { de la production } \\
\text { de } 24 \mathrm{~h}\end{array}$ & $24,70 \%$ & $24,84 \%$ & $25,47 \%$ & $\begin{array}{l}\text { I4 } 455 \\
24,99 \%\end{array}$ \\
\hline
\end{tabular}
au cours de la journée 
La comparaison de la production $6 \mathrm{~h}$.- $\mathrm{I} 2 \mathrm{~h}$. à la production $\mathrm{I} 2 \mathrm{~h}$.- $\mathrm{I} 8 \mathrm{~h}$. montre que les différences observées ne sont pas significatives $(t=0, \mathrm{I} 7$ pour $n=8$ ). La différence moyenne entre ces deux productions peut être évaluée à :

$$
\text { Io } \pm \text { II4 g (seuil } \mathrm{P}=0,05)
$$

Il s'ensuit qu'il est possible, tout en gardant une précision suffisante, de n'effectuer le contrôle de la lactation que sur une demi-journée (6 h.I $2 \mathrm{~h}$.). La production de 24 heures est obtenue en multipliant ce résultat par 4 .

L'erreur faite en assimilant la production de la demi-journée au quart de la production de 24 heures a été calculée : elle est de $86 \pm 56 \mathrm{~g}$ $(\mathrm{P}=0,05)$. Il en résulte, pour le calcul de la production de 24 heures, une erreur moyenne de $\pm 344 \mathrm{~g}$ et une erreur maximum de $\pm 57^{6} \mathrm{~g}$ $(\mathrm{P}=0,05)$.

Soit, en valeur relative, une erreur maximum de 8 p. Ioo.

Cette erreur est du même ordre que celle obtenue à partir du résultat de la journée ( 6 h.-I 8 h.). Nous avons donc adopté pour principe de la méthode, le calcul basé sur la production de la matinée.

\section{V. - NOMBRE DE JOURS DE CONTROLE}

L'espace de temps séparant deux journées de contrôle varie suivant les auteurs: WinOGRADSKY utilisa des intervalles de 5 jours; Bonsma, Schmidt, Hempel, Niwa, Smith, de 7 jours; Hughes et Harte, de Io jours; HENRY et WolL, de $I 7$ jours.

La détermination rigoureuse de l'intervalle à utiliser pour opérer dans des conditions de précision optima nécessiterait une connaissance des résultats de la lactation jour par jour.

Une telle étude n'est malheureusement pas possible en raison de la trop grande perturbation qu'elle entraînerait sur la croissance des animaux. Déjà, BARBER et BRAUDE avaient montré que, dans les conditions où ils opéraient (intervalles entre tétées de 2 à 3 heures), l'influence des opérations de pesée sur le comportement des animaux conduisait à une sous-estimation de la production, lorsque le contrôle durait plus de 24 heures.

Il nous a toutefois été possible d'enregistrer, dans des conditions satisfaisantes, les productions journalières consécutives de deux truies pendant une semaine ( $3^{\mathrm{e}}$ semaine de lactation). Ces productions ont été les suivantes: 
TABLEAU IX

Variations de la production laitière journalière des truies

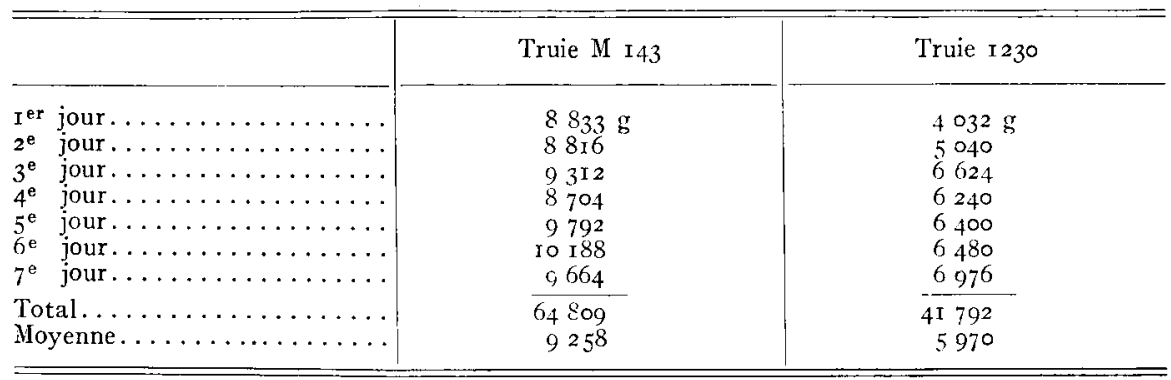

On voit par ces résultats que l'estimation de la production hebdomadaire d'une truie à partir du résultat d'un seul jour de contrôle au milieu de la semaine, peut conduire à faire les erreurs relatives suivantes:

Truie Mr $43 \frac{\text { (Prod. } 4^{\mathrm{e}} \text { jour-Prod. moyenne) } \times 7}{\text { Production de la semaine }} \times$ roo $=6$ p. roo.

Truie I23o $\frac{\text { (Prod. } 4^{\mathrm{e}} \text { jour-Prod. moyenne) } \times 7}{\text { Production de la semaine }} \times$ I00 $=4,5$ p. Ioo.

Ces erreurs ne sont pas négligeables, mais elles montrent que la précision obtenue avec le contrôle hebdomadaire est acceptable (sensiblement du même ordre que celle obtenue avec le contrôle laitier mensuel chez les bovins).

Nous avons donc adopté l'échelonnement suivant dans nos contrôles : premier contrôle 3 jours après la mise-bas, puis un contrôle par semaine à partir de cette date, le dernier contrôle ayant lieu au cours de la $8^{\mathrm{e}}$ semaine de lactation.

\section{VI. - CALCUL DE LA LACTATION. ERREURS}

Il est procédé, par jour de contrôle, à 6 mesures espacées de 75 minutes. La somme de ces six résultats représente la production laitière pour un intervalle de 7 h. 30 . En multipliant cette somme par le coefficient 3,2 , on obtient la production laitière pour 24 heures.

Le report graphique des productions journalières (espacées d'une semaine) permet de dresser une courbe de lactation. L'aire délimitée par cette courbe et l'axe des temps correspond à la production totale pendant la lactation. On peut encore évaluer cette dernière en multipliant par 7 les productions journalières précédentes et en additionnant les productions hebdomadaires ainsi calculées. 
Trente lactations de truies ont déjà pu être enregistrées par nous par cette méthode : la production moyenne journalière par truie a été de $5,9 \mathrm{~kg}$ de lait, ce qui correspond à des lactations de $330 \mathrm{~kg}$ en 8 semaines (pour une moyenne de 9 porcelets par portée).

On trouvera aux figures 2 et 3 , deux exemples de ces lactations.

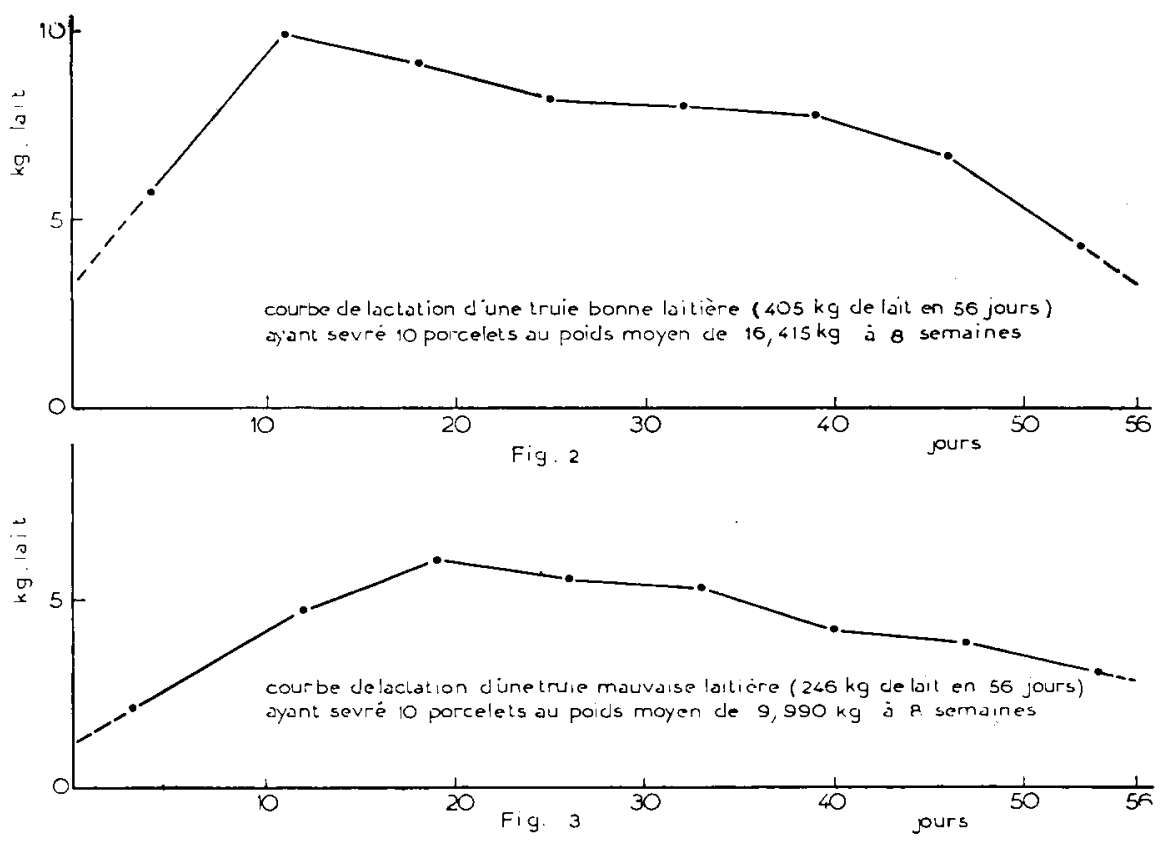

Fig. 2 et 3. - Courbes de lactation de truies

Les erreurs liées à la méthode ne sont pas négligeables, et leur importance conditionne la valeur des résultats. Parmi les plus importantes, il faut retenir :

I0 L'imprécision de la balance et les difficultés de lecture.

Les lectures sont faites avec une précision de ro g. L'erreur possible sur une mesure (nécessitant deux lectures) peut prendre toutes les valeurs entre $+20 \mathrm{~g}$ et $-20 \mathrm{~g}$. On peut démontrer que, dans $95 \mathrm{p}$. Ioo des cas, cette erreur ne dépasse pas \pm ro g. Par ailleurs, les erreurs pouvant affecter différents contrôles se compensent partiellement et au seuil de probabilité $P=0,05$, 1'erreur journalière maximum due à ce facteur ne dépasse pas $130 \mathrm{~g}$ dans le cas de 6 mesures par jour et Igo $\mathrm{g}$ dans le cas de I 2 pesées (portée scindée en deux lots). L'erreur relative qui en résulte est de 2 à $3 \mathrm{p}$. Ioo de la valeur mesurée. 
$2^{\circ}$ Erreurs dues au nombre de mesures par lactation.

Elles ont été calculées précédemment :

- erreur relative due au nombre de mesures par jour

(8 valeurs).

- erreur relative due au nombre de jours de contrôle par lactation $(2$ valeurs $) \ldots \ldots \ldots \ldots \ldots \ldots \ldots \ldots \ldots \ldots \ldots$

$3^{\circ}$ Perte de poids des porcelets au cours des mesures.

Au cours de la tétée, les porcelets perdent légèrement du poids (par respiration et salivation). BARBER et BRAUDE évaluent cette perte à 2-5 $\mathrm{g}$ par porcelet. L'erreur systématique qui en résulte est sensiblement proportionnelle au temps qui s'écoule entre les deux pesées de chaque mesure. Dans les conditions où nous opérons (temps moyen entre les pesées : 3 à 4 minutes), il semble que cette erreur soit faible et inférieure à Io g pour l'ensemble de la portée (Io observations). Elle modifie cependant d'environ 3 p. Ioo (par défaut) la valeur des résultats trouvés.

Il faudrait ajouter à cela certaines erreurs accidentelles : celles occasionnées par les porcelets qui urinent entre les deux pesées d'une mesure. Une telle éventualité est heureusement rare, si l'on opère avec le maximum d'attention et de rapidité, mais lorsqu'elle se produit, il faut appliquer une correction du résultat. Des mesures nous ont conduit à adopter les corrections suivantes pour déjections :

\section{TABLEAU X}

Corrections maxima à appliquer pour déjections des porcelets au cours des mesures

\begin{tabular}{|c|c|c|}
\hline Age des porcelets & Urine & I èces \\
\hline $\begin{array}{l}\text { I semaine } \ldots \ldots \ldots \ldots \ldots \ldots \ldots \ldots \ldots \ldots \ldots \\
3 \text { semaines } \ldots \ldots \ldots \ldots \ldots \ldots \ldots \ldots \ldots \ldots \\
6 \text { semaines } \ldots \ldots \ldots \ldots \ldots \ldots \ldots \ldots\end{array}$ & $\begin{array}{l}10 \underset{g}{g} \\
20 \underset{g}{g} \\
40 \underset{r}{g}\end{array}$ & $\begin{aligned} 5 \mathrm{~g} \\
\mathrm{I} O \mathrm{~g} \\
30 \mathrm{~g}\end{aligned}$ \\
\hline
\end{tabular}

$4^{0}$ Précision générale de la méthode.

Compte tenu de toutes les sources d'erreurs précédentes, l'erreur relative maxima pour le calcul de la lactation est donc de 18 p. Ioo.

Cette erreur peut paraitre importante, mais il est possible d'obtenir une plus grande précision si elle est nécessaire :

a) lorsqu'on ne s'intéresse qu'aux productions journalières (erreur relative $\mathrm{I} 3 \mathrm{p}$. IOO);

b) en utilisant la méthode de référence avec contrôle de toutes les tétées sur 24 heures. La production journalière est alors connue à 6 p. IoO près. 


\section{RÉSÜMÉ}

Une méthode de contrôle des quantités de lait produites par les truies au cours de leur lactation a été étudiée. Cette méthode est basée sur la mesure du gain de poids des porcelets au cours des tétées. On utilise pour cela une balance d'une précision de Io g et d'une portée de $I_{40} \mathrm{~kg}$ permettant de peser des portées entières ou des demi-portées de porcelets. Il est procédé à 8 contrôles, espacés d'une semaine, par lactation; chaque jour de contrôle comprend six mesures de tétées à intervalles de $75 \mathrm{mi}$ nutes et précédées, au début de la matinée, d'une tétée non contrôlée.

Trente lactations ont déjà pu être enregistrées par ce procédé. L'erreur totale à craindre est comprise entre 6 et I 8 p. Ioo de la valeur des résultats selon la technique utilisée.

\section{Reçu pour publication le 29 mars 1956.}

\section{RÉFÉRENCES BIBLIOGRAPHIQUES}

ANDERSEN (K. M.). - Forsogsstationen for svin par Kritiansminde. 2 Beretning Roskilde, I924.

BENGTSSON (S.), (I923-24). - Grissuggans mjölkavkastning och dineras sammansattning. Nordisk Jordbrugsforskning, P. 355.

Barber (R. S.), BRaude (R.), Mitcheli (K. G.) (I954). - Studies on milk production of Large White pigs. J. Agric. Sci., 46, 97.

BERGE (S.), INDREBo (T.) (I953). - Mjolkeproduksjon hos purker. Norges Lantbrukshøgskole, vol. 33, 389 .

Bonsma (F. N.) (I935). - Milk production in Large Black sows. So. African J. Sci., 32, 360 .

CARLYLE (W. L.) (IgO3). - The food requirements of pigs from birth to maturity. Wisc. Agr. Exp. Sta., Bull. I04.

Clausen (H.) et coll. (I952). - (Communication personnelle).

DONALD (H. P.) (I937). - The milk cunsumption and growth of suckling pigs. Emp. J., Exp. Agric., 5, 349.

Dschaparidse (D.) (I936). - Die Milchleistung der schweine. Z. Zucht. B 34,349 .

Filmer (J. F.) (r949). - Observations on the milk yield of sows. Rep. N.Z. Dept. Agric., I948, 49, 87.

Gohren ('T. Von) (I865). - Cité par Barber et Braude. Landw. Vers. Sta. \%, 35I.

Hempei (K.) (I928). - Uber die Milcheistung der Sauen des veredelten Landschweins. Arbeiten der Deutschen Gesellschaft für Zuchtungskunde, $\mathrm{H}$. 37, I9.

Henry (W. A.) et Woll, (F. W.) (I897). - Cité par Barber et Braude, I4th Ann. Rep. Wisc. Agr. Exp. Sta.

Hughes (E. H.) et HART (H. G.) (I935). - Production and composition of sow's milk. J. Nutrit., 9, 3 II.

LALEVIC (D.) (I953). - Study of the milk performance of Morova sows. Arch. poljopr. ovank., 6, I3, I23.

Leroy (A. M.) (I937). - Le Porc, p. I59. Hachette.

NAvratII. (B.) (I955). - Comparaison de la production laitière des truies sui- 
vant le poids des porcelets les $2 \mathrm{I}^{\mathrm{e}}$ et $28^{\mathrm{e}}$ jours. Zivoc. Vjroba. veter. med., I, 43-58.

Niwa ('T.), Ito S. Yokoyama (H.) (I95I). - Studies on the milk secretion of sows. Bull. nat. Inst. A gric. Sci. Japan G; 1, I35.

OrofsSON (N. E. ) et LARSSON (S.) (I930). - Modersuggornas produktion och smagrisarnas utveckling. Centralanstalten Mdd 374 Husdjursavdelningen 68.

Schmidt (J.) et LaUpREcht (G.) (I926). - Uber die Milch der veredelten Landschweinsaüen und ihre Zusammenstezung. Züchtungskunde,p. 50.

Shepperd (J. H.) (Ig29). - N. Dakota tests. Bull. N. Dakota Agr.Exp. Sta., 230.

Smith (D. M.) (I950). - Milk production of the sows. N. Z. Soc. Anim. Prod. Proceed. Ioth Ann. Conf. 89.

Sмiтн (D. M.) (1952). - Milk production in the sow. Proc. N. Z. Soc. Anim. Prod. 12, I02.

Thompson (C. P.) (I93I). - The effect of milk consumption on the growth of suckling pigs. Proc. Amer. Soc. Anim. Prod., 24 th Ann. p. 266.

Weils (W.), BeEson (W. M.), Brady (D. E.) (I940). — Cité par Barber et Braude. Bull. Idaho Agr. Exp. Sta., 236, 9.

WOHLBIER (W.) (I928). - Stoffwechselversuche zum Eiweissansatz bei saugenenden ferkeler. Biochemische Zeitschrift, 202, 29. 